\title{
Lifestyle Stories: Correlating User Information through a Story-Inspired Paradigm
}

\author{
Dana Pavel $^{1}$, Dirk Trossen ${ }^{2}$, Matthias Holweg ${ }^{1}$ \\ ${ }^{1}$ Judge Business School, ${ }^{2}$ Computer Laboratory \\ University of Cambridge \\ Cambridge, UK
}

\begin{abstract}
Lifestyle management area has been developing over the past years due to an increasing individual and societal need for self-awareness. Technologies play an essential role as they can capture, interpret, and visualize various aspects of users' lives. One of the challenges introduced by capturing large and varied amounts of data lays in organizing and displaying it to end users. We present here our novel approach for dealing with such challenge through a story-inspired paradigm. The paper includes a short description of the system design as well as an exploratory study we performed in order to inform and advance our automatic story creation framework.
\end{abstract}

Keywords-Lifestyle monitoring, context-awareness, stories

\section{MOTIVATION AND BACKGROUND}

The role of lifestyle management solutions has become increasingly important as they provide essential support for individuals and healthcare systems through fostering selfawareness [1][2]. However, lifestyles are complex and evolving, therefore systems aimed at this area should not only focus on certain aspects of users' lives but aim to provide a holistic and long-term view [3]. With these goals in mind we created the context-aware lifestyle management experience platform MyRoR [16], able to provide a more comprehensive picture of a user's daily life. Our aim is to support users in identifying not only what happened during the day but also in providing objective clues related to why it might have happened. However, such support requires easy-to-understand interactive interfaces that allow for accessing the available information at both abstract and detailed level. For that,we focused our work on issues around information processing, organization and visualization. Given the large variety of information recorded by our system, we found that using means such as graphs, charts or maps puts too much burden on end users due to the high level of detail. While we employ such means for detailed visualizations in our system, we also looked at novel paradigms for correlating information either for individual use or for sharing. For that, we turned to stories, not only as visualisation means but also as structures for correlating information.

Humans need to socially connect and share their experiences, as noted in [5][6] and, over time, they have used stories to do so [4][7]. Even more, stories are important means for reflecting and rationalising our experiences and emotions [8], both important stages within lifestyle management

${ }^{1}$ This work was performed within the PAL (palproject.org.uk) project, funded by EPSRC through grant number TP/AN072C.

\author{
Vic Callaghan \\ School of Computer Science and Electronic Engineering \\ University of Essex \\ Colchester, UK
}

solutions, as shown in [9]. In [4], a story is defined as " $a$ meaningful collection of events, people, and/or things, which denotes a causal or temporal linkage among them". Various methods exist for computer-mediated storytelling and a very good survey of such work is provided in [10]. Closer to our work are systems that create stories around images taken, such as in [11] and [12]. We found ourselves inspired and motivated by works such as [13] and [14], both focused on capturing and presenting user information centered on emotions.

In our work, we have taken a different approach by focusing on creating a model-based extensible information organization framework where the user information is structured within various contexts grouped along multiple story events (i.e., episodes). The end result is an animated multimedia story object created through a succession of meaningful daily events, displaying the available processed data via images and narratives. As we aimed to better understand how such systems could capture and support end users by reflecting what is important to them, we have not chosen any information as a foreground (e.g., emotions, location) but provided them with the flexibility of deciding. This paper presents the key aspects of our system work as well as a summary of an exploratory study that helped us extend the theoretical framework as well as the system itself. For that, we start with an overview of the information that our platform can gather as well as an overview of how stories are created by processing information and mapping it to multi-media objects. We then present the study and its main findings before concluding with future work.

\section{THE USER INFORMATION IN MYROR}

For most of us, daily activities alternate static with mobile settings, work with play, quiet with noisy, calm with stress. Works such as [2][3][6][8][9][13][14] provide useful insight into lifestyle aspects as well as factors that affect them. In order to address real-life scenarios and capture as many aspects as possible of our lifestyles without the need for intrusive sensors, we decided to focus on three categories of sources, namely wearable sensors, mobile phones, and desktop machines. Figure 1 provides an overview of the information that our platform can collect from these sources. This information is categorized and processed around eight types of contexts:(1) Activity context includes information about what the user was doing as well as measures of activity intensity;(2) Availability context includes information regarding user's availability as well the availability of resources necessary to the user in certain situations;(3) Emotional context (playing an important role in self-reflection [13][14][8]) includes information about 
the user's emotional state, obtained through means ranging from physiological sensing to pattern analysis (e.g., keywords, activities, etc.);(4) Environmental context is determined through environment parameters, especially the ones perceived as having an impact on wellbeing (e.g., ambient noise, weather information, light intensity levels);(5) Mental context refers to the user's interests both in terms of topic (i.e., what the user was interested in) as well as intensity level (i.e., how interested was a user in a certain topic);(6) Social context includes information about the user's social activities, both in the physical and virtual world. Social communication is an important human dimension [5][6] and it plays an important role in wellbeing;(7) Spatial context includes location information at various granularities as well as derived information (e.g., distance or heading);(8) Temporal context, determined through the timestamps recorded with each input source, is essential for correlating information.

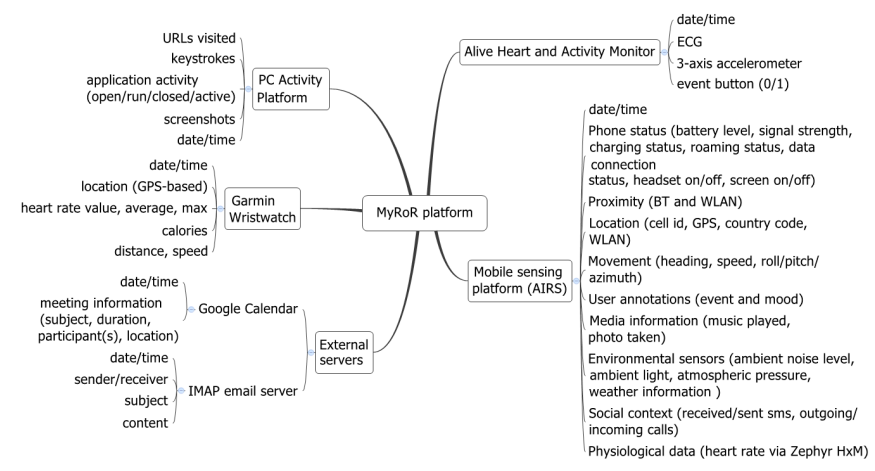

Figure 1. Information and sources used in the MyRoR system

\section{USING STORIES FOR CORRELATING INFORMATION}

Recently, we have seen a growing pool of self-monitoring solutions ${ }^{2}$, mostly using graph or map-based means to display the collected data. While these means provide good detail they are hard to use for bringing together such vast and diverse information. Our stories aim to simplify such process and bring together this data through an engaging multi-media interface. Similar to [4] and [7] the Story Model includes: (1) Setting: defined by parameters such as time, place; (2) Theme: emphasizes one or more types of contexts (e.g., focuses on physical movements or on physiological changes); (3) Characters: the entities that take part in the story (persons or other beings), roles and relationships; (4) Storyline: formed by a sequence of meaningful events, where an event is described in terms of the above context types; (5) Point of view: determines how the story is told based on the intended audience.

Various challenges are involved in creating our stories and they could be summarised through these two main aspects: (1) creating a dynamic and adaptive automatic process, able to decide what information is meaningful enough to be included within a certain user story, how to obtain it and how to present it to end users; (2) enabling users to customize their stories for various purposes such as esthetical reasons, exploring various aspects of available information or sharing. For addressing

\footnotetext{
${ }^{2} \mathrm{~A}$ good collection is available at: http://personalinformatics.org/.
}

these challenges, our research focused on designing a modelbased automated story creation process capable of capturing, processing, organizing and graphically representing user information. The process requires multiple reasoning points for: (1) determining "meaningful" story events; (2) determining the context types for each story event; (3) determining the values for each context type; (4) mapping numeric values to semantic labels for providing human-level meaning; and (5) mapping values onto media that forms the final story.

We considered various methods to determine "meaningful" story events, from end-user driven to fully automatic. In the end, we settled on a mixed approach, based both on user annotating most interesting events during recording (through provided means) and on system-based reasoning (i.e., identifying certain significant changes or event triggers). Once these "meaningful" event triggers are determined, the story events are created by adding a time range around that point (e.g., +/- 5 minutes) allowing us to capture relevant information related to what else was going on around that time. Currently, our stories are created over a specific day and aim to bring together main events from that day's recordings.

For each story event, the available and relevant user information is processed (i.e., filtered, abstracted, correlated) by a rule-based Engine that creates user-level semantic interpretations for information such as heart rate levels, location, environmental conditions (e.g., noise levels, light levels), social interactions and so on. These values are mapped to media such as images and icons, and used to create a text narrative. For processing the multi-variable input data we utilize various algorithms, such as removing outliers, data filtering and aggregation, mean value computation, or determining time-based correlations, such as typing speed (chars/min), phone usage (calls/event), number of messages received over event.

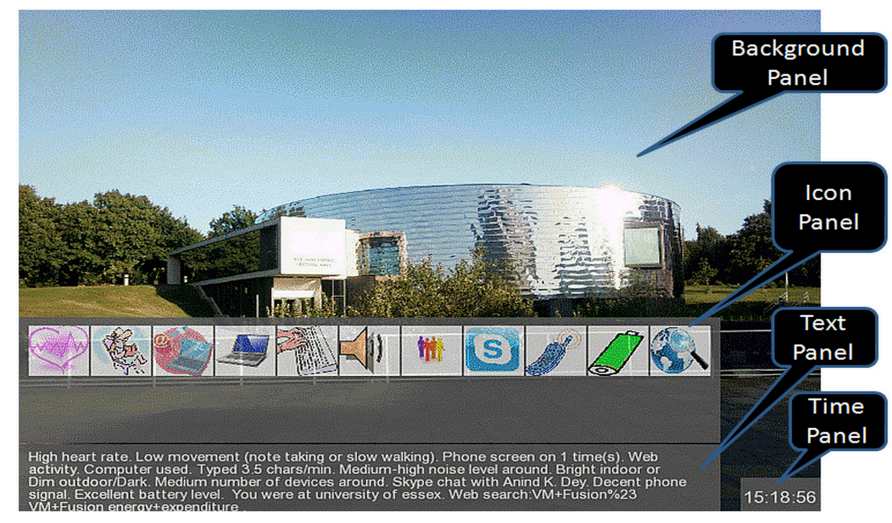

Figure 2. Example of a story event multi-media object

The values computed for each event context are mapped onto graphical elements, as shown in Figure 2, which depicts a story event from a certain day story. The background panel of each event can be customized through user-specific media obtained by mapping from automatically recognized user locations, such as home, university and so on. Such locations can be pre-defined by user based on geocoding or known WiFi SSID. The final story is obtained by assembling the individual event media objects into an animated format. 
An important requirement of our system is that it can evolve by being able to add/remove input sources, processing algorithms and visualizations. The story-based paradigm is very powerful in addressing this requirement as it allows for dynamic changes, personalization through inclusion of usergenerated content, easy sharing, and quick and fun overview of recorded data.

\section{USER-BASED EXPERIMENTS}

Various methods were employed to test and expand our story-based framework, ranging from finding inspiration in existing literature and systems to performing two types of exploratory user-based experiments. The argument for an exploratory focus is provided in [15], and it was particularly apt for our work as we intended to gather further insights and requirements necessary to advance our work. Specifically, we aimed to understand what people find more interesting during the day, how that changes from day to day and from person to person and how stories can provide support for organizing and visualizing lifestyle information. For that, we employed two methods: (1) an online questionnaire focused on capturing self-reflective behaviors vs. attitudes towards the system and its main interface paradigms; (2) hands-on system usage followed by a 2-phased semi-structured interview process for exploring qualitative aspects around the importance of recorded information, experiencing stories and using the system. The results from the online questionnaire were published in [16].

We focus here on the hands-on experiments we performed, involving 6 non-random unpaid volunteers with ages ranging from 30-80 and with a certain interest in self-monitoring. Each participant used the system for recording own data over several days within a normal daily life scenario. User information (as shown in Fig. 1) was gathered through a wearable device for physiological data, a mobile phone running a information gathering platform (see [16]), and a Windows-based activity platform running on one or more of the participant's computers. The participants were instructed to use provided means to annotate during recording what they considered most interesting during their days. Data was collected onto the user's machine and examined during follow-up interviews through various visualization and access means available in our system [16]. The interviews followed a semi-structured interactive and reflective format along a pre-defined set of topics focused on: (1) reasons for annotations; (2) usefulness of information gathered; (3) usefulness of available information correlations; (4) using story-based visualizations for correlating and visualizing information; (5) information sharing aspects; (6) system usages. We used various probing means, such as: (a) reflecting over the annotation process and meaning in relation to their recording context; (b) using the data visualizations to discuss the importance of recorded information and available correlations, and test their role in remembering and reflection; (c) using the story-based concept (Phase1) and developed prototype (Phase2) to collect information on creating, experiencing and sharing stories through discussions around what happened during their days, stimulating memories and self-reflection on meaning and relevance of data. Through this process we collected a wealth of information. We present here a summary of the most interesting findings.
The changing importance of information: The interviews showed that the usefulness of information cannot be detached from the context it was recorded in. For example, within a meeting, important information includes stress levels, participants, ambient noise levels, or room temperature. Within a travel scenario, location becomes more important, together with, e.g., crowd levels, noise and stress levels. The importance of information can also change based on the varying nature of the same event as well as based on certain (chronic or acute) health condition(s) or current goals that take precedence. All participants expected their preferences for what information is recorded and how it is shown to change over time and situation and wanted the system, including the stories, to accommodate such demands. Such support requires a platform approach embedding evolution and adaptation mechanisms.

The reflective power of annotations: While initially used as a way to identify what users considered interesting during recordings, inline annotations eventually became an important part of creating stories, in particular once free-text annotations were added (through mobile phone widgets). Users felt that their annotations added a level of personalization to the system, due to the effort spent in annotating as opposed to only relying on automatically recorded information, especially when used within their stories. Some users also started to utilize the knowledge of what was recorded automatically by the system as well as of how annotations were used later by the system, by capturing with their annotation what was harder to be captured by the system. Hence, annotations were seen as complementing the ability of the system to aid recollection and the annotation became a self-reflective process. All users said they would like their stories to be created by combining their annotations with system intelligence.

Experiencing and creating stories: When presented with their daily stories, all participants valued having a compressed alternative to the detailed visualizations. It was perceived as being easier to understand by bringing all the information together. Comparisons to traditional diaries were made, used for instance to record health-related information, and users expressed their interest in using the system as a digital diary instead. Users also saw stories as providing an information overview compared to studying a variety of detailed visualizations. Stories enabled them to understand the 'essence' quicker because, as one user expressed it, "pictures say more than words". The story-based interface was perceived by some users as a snapshot of oneself, stimulating reflection over the own behavior, therefore fulfilling a central goal of the platform. During both interview phases, participants expressed their views on the story-based interface, some of them being incorporated into the final prototype. Based on such suggestions, icon representations were amended by a text panel (see Figure 2), enabling comprehension of stories through quick glances (via icons) as well as narratives (via the text panel). However, all users expressed the possibility for changing preferences, requiring adaptation of this presentation over time through various customization options. Such options were explored with the participants, both at the level of the graphical interface and in terms of the information shown. While all participants said that they would like their story to feel personal, by being able to have their own icons, pictures 
and so on, they also said they would not like to spend too much time on such customization process. Therefore, they suggested configuration through templates or skins, allowing for customizing what and how information is recorded and shown.

Attitudes towards sharing: An important aspect in sharing stories is that of control, as clearly pointed out by our participants. Control here entails how, what and when something is shared. Some users expressed their interest in being able to select parts of their stories to be shared based on situation/episode (e.g., travelling or being at a party) and not necessarily based on what information should be shown. One user said that, most of the times, he would prefer his shared stories to only include abstracted information, not detailed one (e.g., how much he was typing as opposed to what he was typing). However, sharing detailed information, such as heart rate, movement, location and so on, was seen as useful if it served to make a certain point. This usage as evidence was also mentioned by other users and highlights an important part in aiding recollection and providing support to the user. Various sharing parties, such as existing social networks, focus groups, and healthcare professionals, were mentioned by our users.

\section{CONCLUSIONS AND FUTURE WORK}

In this paper we presented an overview of the model-based story-inspired framework at the center of the MyRoR system we created for addressing lifestyle management scenarios together with main findings from an exploratory study we performed for testing and extending the framework and system. The hands-on experiments were aimed at better understanding how stories should be automatically created in order to incorporate meaningful information and how they should be used for interacting with users. Our multi-media story-based concept was seen by the participants as a very powerful way to encapsulate a vast and varied amount of information, providing an engaging and personal way of access to own recordings. Our experiments showed that stories and lifestyle management solutions in general have to adapt with people, situations and ever-changing health conditions. Hence, we cannot create lifestyle management systems that only focus on a certain type of information since the importance of information changes constantly as users traverse different situations in their lives.

Such insights are useful for informing our future work in extending the story creation framework towards more flexible and dynamic ways of abstracting, personalizing, organizing, prioritizing, and sharing information. For example, we intend to support means for dynamically changing a story focus by selecting a "primary" focus (e.g., stress or physical movement), which would influence what and how supporting information is correlated and shown. One approach to realize such changing focus is through focus templates, as opposed to having the user select the information every time. Another important aspect for our future work is the support for understanding information that spans multiple days or more. Our experiments showed that users would like to extend the daily stories over longer periods. However, such change is not trivial and requires more research, as the length of the stories has to be kept manageable. This also requires the extension of our processing towards identifying patterns and trends over a longer period.
Our experiments highlighted potential use cases, such as identifying stress factors and symptoms. In an ongoing study, we use our system to explore these issues through capturing work-related interactions, such as email, applications used, web activity, in relation to physiological data, user annotations and other context information available through our system. While the study is in its early stages, some findings from our experiments have already been incorporated into our new work.

Another area of interest is that of mobile experiences. Complementing the desktop-focused visualizations of MyRoR, we have recently released the Android-based Storica application. It builds on MyRoR concepts, providing storybased experiences similar to the ones shown in Figure 2 and including map visualizations, media galleries and detailed timelines. Our future work will investigate the applicability of such mobile-centric approach in different usage scenarios.

\section{ACKNOWLEDGMENT}

We would like to thank the participants in our study that volunteered their time and insights for this work.

\section{REFERENCES}

[1] Future Foundation and Friends Provident, "Visions of Britain 2020: Health and Wellbeing," 2010.

[2] Department of Health, "Supporting Self Care-A Practical Option: Diagnostic , Monitoring and Assistive Tools, Devices, Technologies and Equipment to Support Self Care," 2006.

[3] D. T. Wade, "Holistic Health Care: What is it, and how we can achieve it?," 2009.

[4] K. M. Brooks, "Do story agents use rocking chairs? The theory and implementation of one model for computational narrative," in Procs. of the 4th ACM International Conference on Multimedia (MULTIMEDIA '96), pp. 317-328, 1996.

[5] A. H. Maslow, Toward a Psychology of Being, 3rd ed. 2011.

[6] B. Shneiderman, Leonardo's laptop: Human needs and the new computing technologies. Cambridge, MA: MIT Press, 2002.

[7] J. Bruner, "The Narrative Construction of Reality," Critical Inquiry, vol. 18, no. 1, p. 1, Jan. 1991.

[8] G. C. Rosenwald and R. L. Ochberg, Eds., Storied Lives: The Cultural Politics of Self-Understanding. Yale University, 1992.

[9] I. Li, A. K. Dey, and J. Forlizzi, "Understanding My Data, Myself: Supporting Self-Reflection with Ubicomp Technologies," in Procs. of Ubicomp 2011.

[10] A. Nakasone and M. Ishizuka, "Storytelling Ontology Model Using RST," in Procs. of the IEEE/WIC/ACM Int. Conf. on Intelligent Agent Technology, pp. 163-169, Dec. 2006.

[11] L. Doyle, G. Davenport, and D. O’Mahony, "Mobile context-aware stories," in Procs. of the IEEE conf. on Multimedia and Expo, 2002.

[12] D. Byrne, A. Kelliher, and G. J. F. Jones, "Life Editing: Third-Party Perspectives on Lifelog Content," in Procs of CHI 2011, 2011.

[13] P. Sanches, E. K. Vaara, M. Sjölinder, C. Weymann, and K. Höök, "Affective Health - designing for empowerment rather than stress diagnosis," in "Know thyself: monitoring and reflecting on facets of one's life", workshop at CHI 2010.

[14] D. Mcduff, A. Karlson, A. Kapoor, A. Roseway, and M. Czerwinski, "AffectAura: An Intelligent System for Emotional Memory," in Proceedings of CHI2012, May 5-10, Austin, TX, USA, 2012.

[15] G. Ellis and A. Dix, "An explorative analysis of user evaluation studies in information visualisation," in BELIV workshop, 2006.

[16] D. Pavel. V. Callaghan, A.K. Dey, F. Sepulveda, M. Gardner, "The Story of Our Lives: From Sensors to Stories in Self-monitoring Systems," in Procs. of the CEEC'12 conference, Colchester, UK, 2012. 